\title{
Cognitive Profiles and Subtypes of Patients with Mild Cognitive Impairment: Data from a Clinical Follow-Up Study
}

\author{
Kyung Won Park ${ }^{1 \#}$, Eun-Joo Kim², Hwan Joo ${ }^{3}$, Sung-Man Jeon ${ }^{4}$, Seong-Ho Choi ${ }^{5}$, Jay C. Kwon ${ }^{6}$, \\ Byoung Gwon $\mathrm{Kim}^{7}$, Jae Woo Kim ${ }^{1}$ \\ ${ }^{1}$ Department of Neurology, Dong-A University College of Medicine, Dong-A University Medical Center, Busan, South Korea; \\ ${ }^{2}$ Department of Neurology, Pusan National University Hospital, Pusan National University School of Medicine and Medical \\ Research Institute, Busan, South Korea; ${ }^{3}$ Department of Neurology, Busan Medical Center, Busan, South Korea; ${ }^{4}$ Department of \\ Neurology, Bong Seng Memorial Hospital, Busan, South Korea; ${ }^{5}$ Department of Neurology, Wallace Memorial Baptist Hospital, \\ Busan, South Korea; ${ }^{6}$ Department of Neurology, Changwon Fatima Hospital, Changwon, South Korea; ${ }^{7}$ Department of Preventive \\ Medicine, Dong-A University College of Medicine, Busan, South Korea. \\ Email: " neuropark@dau.ac.kr
}

Received May 30 ${ }^{\text {th }}$, 2012; revised June 30 ${ }^{\text {th }}$, 2012; accepted July $17^{\text {th }}, 2012$

\begin{abstract}
Background: Mild cognitive impairment (MCI) is a heterogeneous condition with a variety of clinical outcomes, the presence of which correlates with risk of Alzheimer's disease as well as pre-clinical stages of other dementia subtypes. The aims of this study were to assess the specific patterns of cognitive profiles and to identify changes from baseline to 24 weeks in patients with MCI using detailed neuropsychological testing. Methods: We consecutively recruited 120 MCI patients at baseline according to the Petersen's clinical diagnostic criteria, who were admitted to the Dementia and Memory Clinics. We analyzed patients who fulfilled both inclusion and exclusion criteria for MCI and classified them into four subtypes according to deficits in major cognitive domains; amnestic MCI single domain (aMCI-s), amnestic multiple domain MCI (aMCI-m), non-amnestic single domain MCI (naMCI-s) and non-amnestic multiple domain MCI (naMCI-m). Four groups of MCI were evaluated by a detailed neuropsychological battery test. Results: 83 patients with MCI at the 24-week follow-up were classified into four subtypes. The most frequent subtype was amnestic multi-domain MCI, with the frequency of MCI subtypes as follows: aMCI-s ( $n=21,25.3 \%)$, aMCI-m $(n=53,63.9 \%)$, naMCI-s $(n=5,6.0 \%)$ and naMCI-m $(n=4,4.8 \%)$. In the major cognitive items of the SNSB-D, there were significant changes between the initial and follow-up tests in the domains of language, memory and the frontal/executive function ( $\mathrm{p}<$ 0.05), except for attention, in all MCI patient subtypes. At 24-weeks follow-up, the conversion rate to Alzheimer's disease was $2.4 \%(\mathrm{n}=2)$ from a subtype of amnestic multi-domain MCI. Conclusions: Our study revealed the most frequent subtype of MCI to be multiple domain amnestic MCI, with this subtype having a higher tendency of conversion to Alzheimer's disease.
\end{abstract}

Keywords: Mild Cognitive Impairment; Alzheimer’s Dementia; Neuropsychology; Conversion

\section{Introduction}

The recent increase of an elderly population in developed countries like South Korea has led to a renewed focus on degenerative disorders such as dementia. MCI refers to a mild regression of cognitive functions, in particular memory impairment in patients compared to that of normal people, although the condition is not severe enough to be classified as dementia due to retainment of activeties of daily living, or ADL [1]. According to some epidemiological studies, people with MCI are in a high risk

\footnotetext{
${ }^{*}$ The authors declare no conflicts of interest.

${ }^{\#}$ Corresponding author.
}

group that may progress to Alzheimer's disease [2-4]. Whereas 1 to 2 percent of normal control groups develop dementia annually, 10 to 15 percent of MCI patients develop dementia, in particular, Alzheimer's disease [5]. This condition is clinically important because it is the earliest stage that Alzheimer's disease can be detected and the effects of treatment could therefore be maximized. MCI is a syndrome involving heterogeneous clinical manifestations and diverse causative diseases. The discovery that MCI patients were likely to develop dementia led to the recognition that early diagnosis of MCI patients was crucial.

Furthermore, the classification of MCI patients ac- 
cording to manifestations of cognitive dysfunction resulted in a renewed focus to verify which groups experience a high incidence of Alzheimer's disease among different types of MCI patients [6-8]. Structural or functional brain imaging techniques, such as Magnetic Resonance Imaging (MRI), Positron Emission Tomography (PET) and Single-Photon Emission Computed Tomography (SPECT), were heavily employed for this research, with efforts to verify differences in progression patterns in accordance with the prognoses or types of MCI [6].

A classification of MCI patient subtypes along with detailed neuropsychological tests, in addition to brain imaging techniques, while observing changes in cognitive patterns and prognoses can provide important clues in determining whether to apply pharmacotherapy in a practical clinic setting and make long-term follow-up observations. In a report presenting the international standards for, and classification of MCI [6] suggested that memory disorder was not the only symptom of MCI patients, and that diverse cognitive disabilities may be present, such as visuospatial and language impairment, as well as frontal lobe dysfunction even from an early state of the disease. Therefore, they noted that MCI may be classified into the subtypes of amnestic MCI and nonamnestic MCI, according to whether patients have memory impairment or not. They may also be classified into single and multiple domain disorders, according to the presence of a failing single cognitive domain or of multiple domains. In order to classify patients according to the definitions of the four subtypes of MCI, a working criterion in consideration of age and educational background were used, with neuropsychological test tools representing each cognitive area. Based on the neuropsychological performances, MCI divided into the subtypes: amnestic single domain MCI (aMCI-s), when the patient lacked on the memory function; amnestic multiple domain MCI (aMCI-m), when there were impairments on several cognitive areas, including memory; non-amnestic single domain MCI (naMCI-s), presence of an impairment in another cognitive area, with normal memory; and non-amnestic multiple domain MCI (naMCI$\mathrm{m}$ ), with impairments in more than one cognitive domain, with normal memory.

The frequency and pattern of progression of each subtype of MCI were inconsistent due to the presence of diverse diagnostic criteria, as well as different sampling and assessment methods. The authors performed this study with the following objectives: First, to classify new patients, who visited hospitals from September 2008 to April 2009 that were participating in the study and who were diagnosed with MCI into subtypes (amnestic single domain, amnestic multiple domain, non-amnestic single domain, and non-amnestic multiple domain), and inves- tigate each subtype's frequency and patterns. Second, after six months elapsed, we observed whether the MCI subtypes had changed and whether they had progressed into dementia.

\section{Subjects and Methods}

This was a multi-center, observational study conducted for 24 weeks across 13 hospitals located in Busan Metropolitan City, Gyeongnam Province from September 2008 to February 2010. We performed this study with full approval from the respective Institutional Review Boards of each hospital, after inspection of the clinical trial plan, written explanation for subjects, and after obtaining each subjects' informed consent.

\subsection{Subjects}

We enrolled patients according to working criteria based on the clinical diagnostic criteria for MCI $[5,6]: 1)$ the presence of subjective memory complaint as reported by participants or informants; 2) intact ability to perform activities of normal daily living; 3) normal general cognitive function defined as cognitive performance above the range of 1.0 standard deviation (SD) of normative data in an extensive neuropsychological test; 4) abnormal cognitive function including memory domain for age and education documented by performance of at least 1.0 SD below mean normative data in cognitive tasks; 5) non-demented according to DSM-IV criteria and excluded by fulfilling criteria (2) and (3). The participants included in the present study were 55 to 85 years of age, had not taken acetylcholinesterase inhibitors, memantine or ginkgo bilboa for at least 4 weeks prior to the study initiation, were ambulatory or ambulatory-aided (i.e., walker or cane), had brain MRI or CT scans revealing no clinical evidence of other diseases capable of producing a dementia syndrome, and had a reliable caregiver who met the patient at least once a week and provide the investigator with accurate information. The following exclusion criteria were adopted: 1) major depressive disorder, bipolar disorder, schizophrenia, substance use disorder, or mental retardation according to criteria of the DSM-IV; 2) cerebrovascular disorders, hydrocephalus or intracranial mass, documented by CT or MRI within the past 12 months; 3) abnormalities in serum folate and vitamin B12, syphilis serology, or thyroid hormone levels; 4) history of traumatic brain injury or other neurologic disease; and 5) significant medical problems (e.g. poorly controlled diabetes or hypertension; cancer within the past 5 years; clinically significant hepatic, renal, cardiac or pulmonary disorders).

\subsection{Neuropsychological Tests}

The patients' general cognitive functions were measured 
with the Korean version of the mini-mental state exam (K-MMSE) [9]. The severity of dementia was evaluated with a clinical dementia rating (CDR) [10] and the scores in the six areas of CDR were combined to calculate the sum of boxes (SOB) in each item. Overall severity was expressed with global CDR. All patients underwent neuropsychological tests using a standardized neuropsychological battery called the Seoul Neuropsychological Screening Battery—Dementia Version (SNSB-D) [11,12]. This screening battery contains tests for attention, language, praxis, parietal function, visuoconstructive function, verbal and visual memory, and frontal executive function. The neuropsychological tests were: digit span (forward and backward), the Korean version of the Boston Naming Test (K-BNT) [13], ideomotor praxis, ReyOsterrieth Complex Figure Test (RCFT; copying, immediate and 20-min delayed recall and recognition), the Seoul Verbal Learning Test (SVLT; three learning-free recall trials of 12 words, 20-min delayed recall trial for these 12 items and a recognition test), phonemic and semantic Controlled Oral Word Association Test (COWAT) and the Stroop test (word and color reading of 112 items during a 2-min period). Age-, gender- and educationspecific norms for each test based on 447 normal subjects are available. The scores of these cognitive tests were classified as abnormal when they were below the 16th percentile of the norms for respective age-, gender- and education-matched normal subjects.

\subsection{Classification of MCI Patients According to Subtypes}

In order to classify patients according to the definitions of the four subtypes of MCI, a working criteria in consideration of age and educational background were used, with neuropsychological test tools representing each cognitive area that was specified by the standard established through data research. An experienced neuropsychologist performed the neuropsychological tests on all patients, and based on the results, divided them into the subtypes: 1) amnestic single domain MCI (aMCI-s), when the patient lacked disability in other cognitive areas, except for degraded memory; 2) amnestic multiple domain MCI (aMCI-m), when there were disabilities in other cognitive areas, including memory; 3) non-amnestic single domain MCI (naMCI-s), presence of a disability in another cognitive area, with normal memory; and 4) non-amnestic multiple domain MCI (naMCI-m), with disabilities in more than one cognitive domain, with normal memory.

\subsection{Statistical Analysis}

STATA/SE 11.2 (Stata Corp. 2009, College Station, TX, USA) was used for all statistical analyses, and a two- tailed test was performed with the level of significance set at 0.05 .

A frequency analysis was done of the subtypes, and all data collected through the neuropsychological tests were analyzed using descriptive statistics. Mean values of continuous variables of the data were compared with a paired t-test and non-continuous variables were compared with a chi-square test. When the $\mathrm{P}$ value was less than 0.05 , a difference was considered to be statistically significant. Based on the findings from the neuropsychological tests performed again six months later and the clinicians' own judgments, we calculated the frequency of patients whose MCI progressed to dementia and calculated the rate of conversion of MCI to dementia in each subtype. We ascertained the frequency of each subtype of MCI and the number of MCI patients in each subtype who had changed since the initial diagnosis. In order to verify which cognitive domain underwent the most and least changes, the scores of each of the four cognitive domains (memory, visuospatial ability, linguistic ability and frontal lobe function) were measured, and the scores in the beginning and in the 24th week were compared.

\section{Results}

\subsection{Frequency of Subtypes of MCI at Baseline}

Among the 120 subjects of this study, the follow-up tests of a total of 83 patients were completed. In total, 23 patients dropped out (follow-up loss), 9 patients withdrew their consent to participate in the study, 2 patients were relocated to other hospitals, 1 patient died, 1 patient was excluded due to use of a banned medication and 1 patient was excluded due to the onset of cerebral infarction, all during the follow-up test period. The most common subtype among MCI patients was found to be amnestic multiple domain MCI (aMCI-m). Each subtype's frequency was as follows: aMCI-s is $25.3 \%(\mathrm{n}=21)$, aMCI-m is $63.9 \%(\mathrm{n}=53)$, naMCI-s is $6.0 \%(\mathrm{n}=5)$, and naMCI-m is $4.8 \%(n=4)$ (Table 1$)$.

The rate of male to female patients among the subjects, their average age, and their number of years of education were $34.2 \%$ to $65.8 \%, 68.5 \pm 7.5$ years, and $7.0 \pm 4.3$ years, respectively (Table 1).

\subsection{Changes of Neuropsychological Test Results in Each Subtype at 24 Weeks Follow-Up}

The total score and follow-up score changes of the Seoul Neuropsychological Screening Battery-Dementia Version (SNSB-D) in each subtype are shown in detail in Tables 2-5. After the 24 weeks follow-up period, all MCI patients showed significant improvements in cognitive function on the total score of SNSB-D compared with the baseline assessment $(\mathrm{P}<0.05)$. Furthermore, 
Table 1. Demographic paramters and frequency in patients with MCI according to subtypes.

\begin{tabular}{|c|c|c|c|c|c|c|}
\hline & $\begin{array}{l}\text { Total MCI patients } \\
\quad(\mathrm{n}=120)\end{array}$ & $\begin{array}{c}\text { Amnestic single MCI } \\
\text { patients }(\mathrm{n}=29)\end{array}$ & $\begin{array}{c}\text { Amnestic multiple MCI } \\
\text { patients }(\mathrm{n}=74)\end{array}$ & $\begin{array}{l}\text { Non-amnestic single } \\
\text { MCI patients }(\mathrm{n}=11)\end{array}$ & $\begin{array}{l}\text { Non-amnestic multiple } \\
\text { MCI patients }(n=6)\end{array}$ & $P$ value \\
\hline Female, n (\%) & 79 (65.8) & $22(75.9)$ & $45(60.8)$ & $7(63.6)$ & $5(83.3)$ & 0.397 \\
\hline Age, years & $68.5 \pm 7.5$ & $65.4 \pm 7.8$ & $69.0 \pm 7.2$ & $69.2 \pm 6.9$ & $75.0 \pm 5.9$ & 0.015 \\
\hline Education, years & $7.0 \pm 4.3$ & $6.9 \pm 3.8$ & $7.0 \pm 4.7$ & $7.5 \pm 3.3$ & $6.5 \pm 4.0$ & 0.909 \\
\hline BMI & $24.1 \pm 3.0$ & $24.1 \pm 2.7$ & $24.1 \pm 3.3$ & $24.3 \pm 2.5$ & $23.7 \pm 2.9$ & 0.973 \\
\hline Hypertension, n (\%) & $42(35.0)$ & $10(23.8)$ & $22(52.4)$ & $6(14.3)$ & $4(9.5)$ & 0.145 \\
\hline Diabetes mellitus, n (\%) & $23(19.1)$ & $5(21.7)$ & $14(60.9)$ & $2(8.7)$ & $2(8.7)$ & 0.842 \\
\hline Hyperlipidemia, n (\%) & $13(10.0)$ & $4(30.7)$ & $7(53.9)$ & $0(0.0)$ & $2(15.4)$ & 0.180 \\
\hline Heart disease, n (\%) & $19(15.8)$ & $7(36.8)$ & $9(47.4)$ & $1(5.3)$ & $2(10.5)$ & 0.261 \\
\hline Alcohol drinking, n (\%) & $3(2.5)$ & $0(0.0)$ & $3(4.1)$ & $0(0.0)$ & $0(0.0)$ & 0.431 \\
\hline Smoking, n (\%) & $29(24.4)$ & $4(13.8)$ & $22(31.1)$ & $2(18.1)$ & $1(16.7)$ & 0.557 \\
\hline Depression, n (\%) & $4(3.3)$ & $2(6.0)$ & $2(2.0)$ & $0(0.0)$ & $0(0.0)$ & 0.611 \\
\hline $\begin{array}{l}\text { Family history of } \\
\text { dementia, } \mathrm{n}(\%)\end{array}$ & 23 (19.3) & $3(10.3)$ & $14(19.2)$ & $4(36.4)$ & $2(33.3)$ & 0.230 \\
\hline $\begin{array}{l}\text { ApoE4 genotype } \\
\text { (n = 22), positive, } n\end{array}$ & 8 & 3 & 3 & 1 & 1 & NS \\
\hline
\end{tabular}

Values are presented as number (\%) and mean \pm SD. MCI, mild cognitive impairment; BMI, body mass index.

Table 2. Mean changes in cognitive function on neuropsychological tests in patients with all subtypes of MCI.

\begin{tabular}{|c|c|c|c|}
\hline Outcome measure & Baseline mean (SD) (n = 83) & 24-week F/U mean (SD) $(\mathrm{n}=83)$ & P value \\
\hline MMSE & $26.3(2.3)$ & $26.4(2.4)$ & 0.4238 \\
\hline Attention & $9.1(1.9)$ & $9.4(2.1)$ & 0.1796 \\
\hline Forward & $5.8(1.5)$ & $6.0(1.4)$ & 0.0494 \\
\hline Backward & $3.4(0.9)$ & $3.3(1.1)$ & 0.7313 \\
\hline Language \& related function & $20.0(3.8)$ & $20.8(3.8)$ & 0.0012 \\
\hline Naming (K-BNT) & $10.4(2.9)$ & $10.9(2.7)$ & 0.0279 \\
\hline Calculation & $9.6(2.4)$ & $9.9(2.4)$ & 0.0221 \\
\hline Visuospatial function & $27.6(8.0)$ & $29.1(6.9)$ & 0.0394 \\
\hline Rey figure copy & $27.6(8.0)$ & $29.1(8.0)$ & 0.0394 \\
\hline Memory & $53.8(17.4)$ & $61.9(21.5)$ & $<0.001$ \\
\hline Orientation & $5.6(0.6)$ & $5.6(0.7)$ & 0.7650 \\
\hline Verbal immediate recall & $15.8(5.7)$ & $16.9(6.1)$ & 0.0319 \\
\hline Verbal delayed recall & $3.5(2.4)$ & $5.1(2.8)$ & 0.0000 \\
\hline Verbal recognition index & $7.4(2.1)$ & $7.6(2.6)$ & 0.5153 \\
\hline Visual immediate/delayed recall & $15.2(11.7)$ & $20.2(13.8)$ & $<0.001$ \\
\hline Visual recognition & $6.2(2.4)$ & $6.5(2.5)$ & 0.2782 \\
\hline Frontal/executive & $44.4(10.2)$ & $46.3(9.8)$ & 0.0039 \\
\hline Impersistence & $3.0(0.0)$ & $3.0(0.0)$ & N/A \\
\hline Contrasting program & $2.6(0.7)$ & $2.7(0.5)$ & 0.1316 \\
\hline Go-no-go test & $2.0(1.0)$ & $2.3(0.8)$ & 0.0011 \\
\hline Fist-edge-palm & $2.7(0.5)$ & $2.7(0.5)$ & 0.8587 \\
\hline Luria loop & $2.6(0.9)$ & $2.8(0.7)$ & 0.0356 \\
\hline Word fluency-animal & $13.1(3.9)$ & $12.8(3.6)$ & 0.3324 \\
\hline Word fluency-phonemic & $5.6(3.9)$ & $5.9(3.4)$ & 0.4209 \\
\hline Stroop test-color & $12.8(4.5)$ & $14.0(4.7)$ & 0.0010 \\
\hline SNSB-D & 154.9 (32.6) & 167.4 (35.9) & $<0.001$ \\
\hline CDR sum of box & $1.3(0.8)$ & $1.2(0.8)$ & 0.0357 \\
\hline IADL & $3.5(3.6)$ & $2.7(3.8)$ & 0.0362 \\
\hline NPI & $4.0(8.3)$ & $3.8(8.0)$ & 0.7711 \\
\hline Geriatric depression scale & $16.9(6.8)$ & $15.6(7.0)$ & 0.0555 \\
\hline
\end{tabular}

MCI, mild cognitive impairment; MMSE, Mini-Mental State Examination; SNSB-D, Seoul Neuropsychological Screening Battery-Dementia Version; K-BNT, Korean Boston Naming Test; CDR, Clinical Dementia Rating; IADL, Instrumental Activities of Daily Living; NPI, Neuropsychiatric inventory. 
Table 3. Mean changes in cognitive function on neuropsychological tests in patients with aMCI-s.

\begin{tabular}{|c|c|c|c|}
\hline \multirow{2}{*}{ Outcome measure } & \multicolumn{3}{|c|}{ aMCI-s $(\mathrm{n}=21)$} \\
\hline & Baseline mean (SD) & 24-week F/U mean (SD) & $P$ value \\
\hline MMSE & $26.9(1.7)$ & $27.2(1.7)$ & 0.4127 \\
\hline Attention & $9.8(2.4)$ & $10.2(2.2)$ & 0.4123 \\
\hline Forward & $6.1(1.7)$ & $6.4(1.4)$ & 0.4361 \\
\hline Backward & $3.7(0.9)$ & $3.8(1.3)$ & 0.7152 \\
\hline Language \& related function & $21.0(3.4)$ & 21.7 (3.5) & 0.0784 \\
\hline Naming (K-BNT) & $11.1(2.1)$ & $11.5(2.5)$ & 0.3081 \\
\hline Calculation & $9.8(2.5)$ & $10.1(2.2)$ & 0.0571 \\
\hline Visuospatial function & $31.8(4.8)$ & $31.4(4.5)$ & 0.8171 \\
\hline Rey figure copy & $31.8(4.8)$ & $31.5(4.5)$ & 0.8171 \\
\hline emory & $57.8(22.2)$ & $67.9(22.0)$ & 0.0063 \\
\hline Orientation & $5.7(0.6)$ & $5.6(0.7)$ & 0.5402 \\
\hline Verbal immediate recall & $14.9(5.7)$ & 16.8 (6.80.) & 0.1290 \\
\hline Verbal delayed recall & $3.2(2.5)$ & $5.3(2.8)$ & 0.0022 \\
\hline Verbal recognition index & $7.1(1.9$ & $7.9(2.3)$ & 0.0725 \\
\hline Visual immediate/delayed recall & $20.2(15.9)$ & $25.1(13.7)$ & 0.0539 \\
\hline Visual recognition rey & $6.6(2.2)$ & $7.2(2.4)$ & 0.3000 \\
\hline Frontal/executive & 50.7 (9.9) & $51.4(9.3)$ & 0.5190 \\
\hline Impersistence & $3.0(0.0)$ & $3.0(0.0)$ & - \\
\hline Contrasting program & $2.8(0.5)$ & $2.9(0.4)$ & 0.7477 \\
\hline Go-no-go test & $2.0(0.9)$ & $2.5(0.7)$ & 0.0466 \\
\hline Fist-edge-palm & $2.8(0.5)$ & $2.8(0.5)$ & 1.0000 \\
\hline Luria loop & $2.7(0.7)$ & $3.0(0.0)$ & 0.0829 \\
\hline Word fluency-animal & $14.2(4.5)$ & $14.0(3.8)$ & 0.6799 \\
\hline Word fluency-phonemic & $6.4(3.8)$ & $6.7(3.8)$ & 0.6708 \\
\hline Stroop test-color & $16.7(3.4)$ & $16.7(4.0)$ & 0.9157 \\
\hline SNSB-D & $171.0(33.8)$ & 152.7 (32.6) & 0.0127 \\
\hline CDR sum of box & $1.1(0.6)$ & $1.0(0.6)$ & 0.0829 \\
\hline IADL & $2.8(3.9)$ & $1.6(2.7)$ & 0.0146 \\
\hline NPI & $4.0(10.9)$ & $4.0(12.1)$ & 0.9114 \\
\hline Geriatric depression scale & $19.0(5.9)$ & $16.4(8.6)$ & 0.0494 \\
\hline
\end{tabular}

aMCI-s, single domain amnestic mild cognitive impairment; MMSE, Mini-Mental State Examination; SNSB-D, Seoul Neuropsychological Screening Battery-Dementia Version; K-BNT, Korean Boston Naming Test; CDR, Clinical Dementia Rating; IADL, Instrumental Activities of Daily Living; NPI, Neuropsychiatric inventory.

patients with each subtype of MCI showed significant improvement on the total score of SNSB-D compared with the baseline assessment $(\mathrm{P}<0.05)$. The mean differences of total scores of SNSB-D in patients with MCI were as follows; entire MCI $(12.5 \pm 18.7)$, aMCI-s (11.7 \pm 19.6), aMCI-m (12.6 \pm 19.9), naMCI-s (13.0 \pm 9.7), naMCI-m (15.3 \pm 5.3$)$. The other changes in the cognitive outcome measures are shown in detail in Tables 2-5.

\subsection{Progression of Subjects with MCI}

In the follow-up test after 24 weeks, two MCI patients (2.4\%) progressed to Alzheimer's disease; while their subtype during the initial test was aMCI-m. No patient progressed to a type of dementia other than Alzheimer's disease. The number of MCI patients who underwent conversion to a normal condition was five (6\%) and their MCI subtypes were aMCI-s (1 person), aMCI-m (3 persons) and naMCI-s (1 person). After 24 weeks, the frequencies of each subtype were as follows: aMCi-s was $25.5 \%$, aMCI-m was $42.1 \%$, naMCI-s was $16.9 \%$, and naMCI-m was $7.2 \%$.

\section{Discussion}

Previous research on MCI has revealed that it not only consists of the amnestic type, but rather a range of dif- 
Table 4. Mean changes in cognitive function on neuropsychological tests in patients with aMCI-m.

\begin{tabular}{|c|c|c|c|}
\hline \multirow{2}{*}{ Outcome measure } & \multicolumn{3}{|c|}{ aMCI-m (n = 53) } \\
\hline & Baseline mean (SD) & 24-week F/U mean (SD) & P value \\
\hline MMSE & $25.9(2.4)$ & $26.0(2.7)$ & 0.6770 \\
\hline Attention & $9.0(1.8)$ & $9.1(2.0)$ & 0.3503 \\
\hline Forward & $5.7(1.4)$ & $6.0(1.4)$ & 0.0795 \\
\hline Backward & $3.2(0.9)$ & $3.1(1.1)$ & 0.5603 \\
\hline Language \& related function & $19.3(3.9)$ & 20.1 (3.9) & 0.0159 \\
\hline Naming (K-BNT) & $9.9(3.2)$ & $10.4(2.7)$ & 0.0751 \\
\hline Calculation & $9.4(2.6)$ & $9.6(2.5)$ & 0.1593 \\
\hline Visuospatial function & $25.9(8.9)$ & $28.0(7.9)$ & 0.0287 \\
\hline Rey figure copy & $25.9(8.9)$ & $28.0(7.8)$ & 0.0287 \\
\hline Memory & $50.5(15.1)$ & $57.7(21.2)$ & 0.0004 \\
\hline Orientation & $5.5(0.6)$ & $5.6(0.8)$ & 0.3742 \\
\hline Verbal immediate recall & $16.0(5.6)$ & $16.5(5.6)$ & 0.3862 \\
\hline Verbal delayed recall & $3.4(2.4)$ & $4.9(2.8)$ & 0.0000 \\
\hline Verbal recognition index & $7.3(2.3)$ & $7.2(2.7)$ & 0.6062 \\
\hline Visual immediate/delayed recall & $12.2(9.3)$ & $17.4(13.6)$ & 0.0009 \\
\hline Visual recognition rey & $6.0(2.6)$ & $6.2(2.5)$ & 0.6641 \\
\hline Frontal/executive & $41.9(9.6)$ & $44.1(9.6)$ & 0.0126 \\
\hline Impersistence & $3.0(0.0)$ & $3.0(0.0)$ & - \\
\hline Contrasting program & $2.5(0.8)$ & $2.7(0.5)$ & 0.1066 \\
\hline Go-no-go test & $1.9(1.0)$ & $2.2(0.9)$ & 0.0177 \\
\hline Fist-edge-palm & $2.7(0.5)$ & $2.8(0.5)$ & 0.6590 \\
\hline Luria loop & $2.4(1.0)$ & $2.7(0.9)$ & 0.0963 \\
\hline Word fluency-animal & $12.4(3.6)$ & $12.3(3.6)$ & 0.7112 \\
\hline Word fluency-phonemic & $5.4(3.8)$ & $5.5(3.1)$ & 0.7719 \\
\hline Stroop test-color & $11.5(4.2)$ & $13.0(4.9)$ & 0.0053 \\
\hline SNSB-D & $146.5(31.0)$ & 159.0 (36.9) & 0.0000 \\
\hline CDR sum of box & $1.5(0.9)$ & $1.3(0.8)$ & 0.1212 \\
\hline IADL & $3.8(3.7)$ & $3.3(4.3)$ & 0.3476 \\
\hline NPI & $4.5(7.8)$ & $4.0(6.4)$ & 0.5156 \\
\hline Geriatric depression scale & $16.1(7.1)$ & $1537(6.4)$ & 0.6306 \\
\hline
\end{tabular}

aMCI-m, multiple domain amnestic mild cognitive impairment; MMSE, Mini-Mental State Examination; SNSB-D, Seoul Neuropsychological Screening Battery-Dementia Version; K-BNT, Korean Boston Naming Test; CDR, Clinical Dementia Rating; IADL, Instrumental Activities of Daily Living; NPI, Neuropsychiatric inventory.

ferent subtypes. As proposed by Winbald et al. [6], MCI can be classified into four different types, according to disabilities in domains identified by neuropsychological tests. We consider that each subtype has different causes and prognoses. Recently published research has reported a classification of MCI patients into subtypes according to neuropsychological tests with the observation of each subtype's clinical manifestations and prognoses. This resulted in the finding that MCI was not a pre-stage of Alzheimer's disease, but rather consisted of a group of heterogeneous diseases with various kinds of prognoses $[1,14]$. According to further research, aMCI-m is likely to progress to Alzheimer's disease, while naMCI-s or
naMCI-m are likely to progress to vascular dementia, frontotemporal dementia or dementia with Lewy bodies [1,15].

To our knowledge, this is the first regional study of its kind performing neuropsychological assessments on 120 MCI patients, calculating the frequency of each subtype of MCI, observing changes in each subtype's frequency after a 24-week follow-up test and analysis of the changes in the cognitive functions of each type of patient. We found that the most common subtype of MCI was aMCI-m (63.9\%), followed by aMCI-s (25.3\%), naMCI-s (6.0\%), and naMCI-m (4.8\%). Our results differ from that of a prior study (Fischer et al., 2007), where the 
Table 5. Mean changes in cognitive function on neuropsychological tests in patients with naMCI.

\begin{tabular}{|c|c|c|c|}
\hline \multirow{2}{*}{ Outcome measure } & \multicolumn{3}{|c|}{$\operatorname{naMCI}(\mathrm{n}=9)$} \\
\hline & Baseline mean (SD) & 24-week F/U mean (SD) & P value \\
\hline MMSE & $26.8(2.5)$ & $26.9(1.8)$ & 0.8695 \\
\hline \multicolumn{4}{|l|}{ Attention } \\
\hline Forward & $5.1(1.3)$ & $5.4(1.7)$ & 0.4714 \\
\hline Backward & $3.5(0.5)$ & $3.4(0.5)$ & 0.3466 \\
\hline \multicolumn{4}{|l|}{ Language \& related function } \\
\hline Naming (K-BNT) & $11.3(2.4)$ & $11.7(2.6)$ & 0.3466 \\
\hline Calculation & $10.7(1.3)$ & $11.1(1.2)$ & 0.1950 \\
\hline \multicolumn{4}{|l|}{ Visuospatial function } \\
\hline Rey figure copy & $28.0(4.4)$ & $29.8(3.4)$ & 0.3321 \\
\hline \multicolumn{4}{|l|}{ Memory } \\
\hline Orientation & $5.7(0.5)$ & $5.6(0.5)$ & 0.5943 \\
\hline Verbal immediate recall & $16.8(6.1)$ & $19.3(7.4)$ & 0.0171 \\
\hline Verbal delayed recall & $5.0(1.7)$ & $6.1(2.8)$ & 0.0619 \\
\hline Verbal recognition index & $8.6(1.1)$ & $9.3(1.3)$ & 0.1411 \\
\hline Visual immediate/delayed recall & $21.6(6.6)$ & $25.6(10.3)$ & 0.1577 \\
\hline Visual recognition rey & $6.4(2.1)$ & $6.8(1.8)$ & 0.6454 \\
\hline \multicolumn{4}{|l|}{ Frontal/executive } \\
\hline Contrasting program & $2.6(1.0)$ & $2.7(0.7)$ & 0.7995 \\
\hline Go-no-go test & $2.3(1.0)$ & $2.6(0.7)$ & 0.3466 \\
\hline Fist-edge-palm & $2.7(0.7)$ & $2.6(0.7)$ & 0.3466 \\
\hline Luria loop & $3.0(0.0)$ & $2.9(0.3)$ & 0.3466 \\
\hline Word fluency-animal & $14.8(3.5)$ & $13.2(3.3)$ & 0.2021 \\
\hline Word fluency-phonemic & $4.9(4.4)$ & $6.0(4.2)$ & 0.1786 \\
\hline Stroop test-color & $11.1(3.4)$ & $14.0(2.8)$ & 0.0064 \\
\hline SNSB-D & $167.2(22.1)$ & $181.2(21.2)$ & 0.0006 \\
\hline CDR sum of box & $0.8(0.3)$ & $0.8(0.4)$ & 0.5943 \\
\hline IADL & $3.2(2.0)$ & $1.9(1.8)$ & 0.2572 \\
\hline NPI & $0.3(1.0)$ & $2.2(3.5)$ & 0.1276 \\
\hline Geriatric depression scale & $17.0(7.0)$ & $13.4(7.0)$ & 0.1354 \\
\hline
\end{tabular}

naMCI, non-amnestic mild cognitive impairment; F/U, follow-up; MMSE, Mini-Mental State Examination; SNSB-D, Seoul Neuropsychological Screening Battery-Dementia Version; K-BNT, Korean Boston Naming Test; CDR, Clinical Dementia Rating; IADL, Instrumental Activities of Daily Living; NPI, Neuropsychiatric inventory.

frequency of subtypes of MCI patients were 48 out of 141 (34\%) patients with aMCI and 93 out of 141 (66\%) patients with naMCI. The frequency of MCI subtypes in further studies showed that the prevalence of each subtype of MCI in research, where the subjects were from the general population, was different to that of the current study $[16,17]$. They reported that the nonamnestic MCI type was as frequent as the amnestic MCI type, while the single domain amnestic MCI was more prevalent than multiple domain amnestic MCI. Another study reported that the prevalence of aMCI and naMCI was around $9 \%$ and $15 \%$, respectively. The overall rate of naMCI was even higher than that of aMCI in the community-based study [18].
One of the main reasons for the different rates in the frequency of MCIs may be due to the difference between hospital-based studies and community-based epidemiological studies. The other reason for differing rates of frequency in MCI subtypes is a dissimilar adaptation of operational criteria for defining MCI. Our study defined memory impairment in the Seoul Verbal Learning Test (SVLT) as 1 standard deviation (SD) below age- and education-matched control subjects, unlike other studies [5,8], therefore a larger number of patients were classified into the aMCI subtype. It is also possible that cultural differences between Korea and other countries affected the scores; for example in the United States, visuospatial impairment displayed during driving, or disabili- 
ties in planning or decision making, may have been considered important in addition to memory impairment. Furthermore, this study included cases where patients' guardians or informants reported memory impairment even without subjective memory complaints from the patients, which resulted in a higher rate of MCI cases relative to those of other studies.

During the 24 weeks follow-up period, all patients of MCI with each subtype of MCI showed significant improvements in cognitive function on the total score of the SNSB-D compared with the baseline assessment. In the major cognitive items of the SNSB-D, there was significant change between the initial and the follow-up assessments in the domains of language, memory and the frontal/executive function in all subtypes of MCI. This result is probably due to a relatively large number of patients with high MMSE scores and mild stage of patients being included, and learning effect may have resulted during the relatively short follow-up period. The condition of depression may also have had an influence on the test scores, given that the patients with high depression scores in the initial tests showed improvement in the follow-up tests.

Our study showed that two MCI patients (2.4\%) progressed to Alzheimer's disease; their subtype during the initial test was aMCI-m. No patient progressed to other types of dementia other than Alzheimer's disease. A 3-year follow-up research study reported that rates of conversion to Alzheimer's disease for the MCI subtypes were $38 \%$ for amnestic MCI, 20\% for non-amnestic MCI and $16 \%$ for amnestic multiple domain MCI [7]. The other long-term follow-up study revealed a conversion rate to $\mathrm{AD}$ at $49 \%$ for amnestic MCI and $27 \%$ for nonamnestic MCI [8]. Our study did not show in-depth conversion rates due to a shorter follow-up period. However, our results show a tendency for progression to AD from amnestic MCI rather than non-amnestic MCI.

We identified some shortcomings in our study. First, although it was a prospective follow-up study, we did observe changes in the MCI patients' subtypes and their cognitive functions during the short period of 24 weeks, therefore making it difficult to form a precise evaluation of the patients' disease progression or an estimation of the annual rate of conversion from MCI to dementia. Second, the number of patients in each subtype was unevenly distributed, making it difficult to determine true statistical significance. Also, differences in levels of apolipoprotein E4 were not obtained from all patients, nor the analysis of differences in MCI in accordance with the existence or non-existence of cerebrovascular lesions, such as white matter changes or lacunar infarction.

In conclusion, we were able to verify that the SNSB-D is a useful tool to classify MCI subtypes and follow their progression in detail, suggesting that the routine classifi- cation of MCI into subtypes and observation of progression may be conducive to predicting a transition of MCI to Alzheimer's disease or other types of dementia. In our study, aMCI-m was the most common subtype among the four subtypes of MCI, and its rate of conversion into Alzheimer's disease was statistically significant. For a more comprehensive investigation, it will be necessary to perform further prospective multi-center research for a period of a few years, with many hundreds of patients as subjects, in region-based cohort studies as well as hospital-based cohort studies.

\section{Acknowledgements}

This study was supported by a grant from the Korea Healthcare Technology R\&D Project, Ministry of Health and Welfare, Republic of Korea (A102065) and Eisai Korea Inc.

\section{REFERENCES}

[1] R. C. Petersen, R. Doody, A. Kurz, R. C. Mohs, J. C. Morris, P. V. Rabins, et al., "Current Concepts in Mild Cognitive Impairment,” Archives of Neurology, Vol. 58, No. 12, 2001, pp. 1985-1992.

doi:10.1001/archneur.58.12.1985

[2] H. Amieva, L. Letenneur, J. F. Dartigues, I. RouchLeroyer, C. Sourgen, F. D’Alchee-Biree, et al., “Annual Rate and Predictors of Conversion to Dementia in Subjects Presenting Mild Cognitive Impairment Criteria Defined According to a Population-Based Study,” Dementia and Geriatric Cognitive Disorders, Vol. 18, No. 1, 2004, pp. 87-93. doi:10.1159/000077815

[3] P. Alexopoulos, T. Grimmer, R. Perneczky, G. Domes and A. Kurz, "Progression to Dementia in Clinical Subtypes of Mild Cognitive Impairment," Dementia and Geriatric Cognitive Disorders, Vol. 22, No. 1, 2006, pp. 27-34. doi:10.1159/000093101

[4] The Canadian Study of Health and Aging Working Group, "The Incidence of Dementia in Canada," Neurology, Vol. 55, No. 1, 2000, pp. 66-73. doi:10.1212/WNL.55.1.66

[5] R. C. Petersen, G. E. Smith, S. C. Waring, R. J. Ivnik, E. G. Tangalos and E. Kokmen, "Mild Cognitive Impairment: Clinical Characterization and Outcome," Archives of Neurology, Vol. 56, No. 3, 1999, pp. 303-308. doi:10.1001/archneur.56.3.303

[6] B. Winblad, K. Palmer, M. Kivipelto, V. Jelic, L. Fratiglioni, L. O. Wahlund, et al., "Mild Cognitive Impairment-Beyond Controversies, Towards a Consensus: Report of the International Working Group on Mild Cognitive Impairment,” Journal of Internal Medicine, Vol. 256, No. 3, 2004, pp. 240-246. doi:10.1111/j.1365-2796.2004.01380.x

[7] F. Maioli, M. Coveri, P. Pagni, C. Chiandetti, C. Marchetti, R. Ciarrocchi, et al., "Conversion of Mild Cognitive Impairment to Dementia in Elderly Subjects: A Preliminary Study in a Memory and Cognitive Disorder Unit,” Archives of Gerontology and Geriatrics, Vol. 44, 
No. 1, 2007, pp. 233-241. doi:10.1016/j.archger.2007.01.032

[8] P. Fischer, S. Jungwirth, S. Zehetmayer, S. Weissgram, S. Hoenigschnabl, E. Gelpi, et al., "Conversion from Subtypes of Mild Cognitive Impairment to Alzheimer Dementia,” Neurology, Vol. 68, No. 4, 2007, pp. 288-291. doi:10.1212/01.wnl.0000252358.03285.9d

[9] Y. Kang, D. L. Na and S. Hahn, "A Validity Study on the Korean Mini-Mental State Examination(K-MMSE) in Dementia Patients,” Journal of the Korean Neurological Association, Vol. 15, 1997, pp. 300-308.

[10] J. C. Morris, "The Clinical Dementia Rating (CDR): Current Version and Scoring Rules,” Neurology, Vol. 43, No. 11, 1993, pp. 2412-2414. doi:10.1212/WNL.43.11.2412-a

[11] Y. Kang and D. L. Na, "Seoul Neuropsychological Screening Battery," Human Brain Research \& Consulting Co., Seoul, 2003.

[12] H. J. Ahn, J. Chin, A. Park, B. H. Lee, M. K. Suh, S. W. Seo, et al., "Seoul Neuropsychological Screening Battery-Dementia Version (SNSB-D): A Useful Tool for Assessing and Monitoring Cognitive Impairments in Dementia Patients,” Journal of Korean Medical Science, Vol. 25, No. 7, 2010, pp. 1071-1076. doi:10.3346/jkms.2010.25.7.1071

[13] H. Kim and D. L. Na, "Normative Data on the Korean Version of the Boston Naming Test," Journal of Clinical and Experimental Neuropsychology, Vol. 21, No. 1, 1999, pp. 127-133. doi:10.1076/jcen.21.1.127.942

[14] J. T. Tschanz, K. A. Welsh-Bohmer, C. G. Lyketsos, C. Corcoran, R. C. Green, K. Hayden, et al., "Conversion to Dementia from Mild Cognitive Disorder: The Cache County Study,” Neurology, Vol. 67, No. 2, 2006, pp. 229234. doi:10.1212/01.wnl.0000224748.48011.84

[15] M. Zanetti, C. Ballabio, C. Abbate, C. Cutaia, C. Vergani and L. Bergamaschini, "Mild Cognitive Impairment Subtypes and Vascular Dementia in Community-Dwelling Elderly People: A 3-Year Follow-Up Study,” Journal of the American Geriatrics Society, Vol. 54, No. 4, 2006, pp. 580-586. doi:10.1111/j.1532-5415.2006.00658.x

[16] A. Busse, A. Hensel, U. Guhne, M. C. Angermeyer and S. G. Riedel-Heller, "Mild Cognitive Impairment: LongTerm Course of Four Clinical Subtypes,” Neurology, Vol. 67, No. 12, 2006, pp. 2176-2185. doi:10.1212/01.wnl.0000249117.23318.e1

[17] J. J. Manly, M. X. Tang, N. Schupf, Y. Stern, J. P. Vonsattel and R. Mayeux, "Frequency and Course of Mild Cognitive Impairment in a Multiethnic Community," Annals of Neurology, Vol. 63, No. 4, 2008, pp. 494-506. doi:10.1002/ana.21326

[18] S. Jungwirth, S. Weissgram, S. Zehetmayer, K. H. Tragl and P. Fischer, "VITA: Subtypes of Mild Cognitive Impairment in a Community-Based Cohort at the Age of 75 Years," International Journal of Geriatric Psychiatry, Vol. 20, No. 5, 2005, pp. 452-458. doi:10.1002/gps.1311 